\title{
Facteurs paternels influençant le développement embryonnaire
}

\author{
J. PARINAUd
}

Laboratoire de Fécondation In Vitro, CHU La Grave, 31052 Toulouse

\section{RESUME}

L'influence des facteurs spermatiques sur le développement embryonnaire dans l'espèce humaine a été démontré par la constatation d'une moindre qualité des embryons obtenus en Fécondation In Vitro en cas d'infertilité masculine par rapport aux stérilités tubaires. Les facteurs potentiellement impliqués sont multiples. C'est ainsi que le centrosome du zygote, centre organisateur des microtubules et jouant ainsi un rôle essentiel dans les mitoses, est exclusivement d'origine spermatique et certains échecs de FIV ont pu être reliés à des anomalies du centrosome spermatique. Un facteur cytosolique du spermatozoïde, l'oscilline, est responsable de l'activation ovocytaire post fécondation et pourrait être impliqué dans les retards de clivage constatés dans les anomalies du sperme. Les anomalies chromosomiques constatées dans certaines oligospermies sévères pourraient être responsables d'arrêts de développement embryonnaire. Enfin, les phénomènes d'empreintes génomiques, se produisant lors de la spermatogénèse, pourraient être imparfaits dans les dysfonctionnements testiculaires ce qui conduirait à l'obtention d'embryons ayant des capacités de développement réduites. Toutefois, l'absence d'influence de facteurs spermatiques sur les résultats de l'ICSI tend à montrer que cette technique contourne le problème rencontré en FIV. Dans ces conditions, le facteur le plus probablement impliqué semble être l'activation ovocytaire.

Mots clés : spermatozoïde, embryon, centrosome, oscilline, génétique.

La plupart des études effectuées sur les aspects spermatiques de l'interaction gamétique se sont focalisées sur la fécondation et pour beaucoup d'auteurs l'impact de l'infertilité masculine se limite à l'obtention ou non d'embryons. Toutefois, des études récentes ont montré que la qualité du sperme pouvait jouer un rôle important dans le développement embryonnaire. Les mécanismes potentiellement mis en jeu sont multiples et certains semblent pouvoir être contournés par l'injection intracytoplasmique (ICSI). Dans cet article nous faisons une revue des données cliniques et fondamentales disponibles sur le sujet.

\section{DONNEES CLINIQUES}

\section{Fécondation In Vitro}

La fécondation in vitro est un bon modèle d'étude de l'influence des facteurs masculins sur le développement embryonnaire, puisqu'elle permet d'une part de faire la part des échecs de fécondations et des défauts de développement embryonnaire 
dans les infertilités masculines, et d'autre part d'évaluer la qualité des premiers stades de la croissance de l'embryon.

C'est ainsi que nous avons montré [1] que, en fécondation in vitro, la qualité morphologique des embryons était altérée et que le taux de nidation était diminué dans les oligo-asthéno-tératospermies sévères. En effet, lorsque l'on étudie la qualité morphologique des embryons au stade 4 cellules (classée de 1 à 3 ) en fonction des indications, il s'avère que le pourcentage de transferts d'embryons de très bonne qualité (groupe $3: 4$ blastomères de taille identique et absence d'exsudats cytoplasmiques) est significativement plus élevé dans les stérilités d'origine tubaire que dans les stérilités masculines (Tableau 1). La situation est inverse pour les transferts d'embryons de moins bonne qualité morphologique (groupe 1 : blastomères de tailles inégales et nombreux exsudats cytoplasmiques).
Si l'on regarde de façon plus détaillée les paramètres spermatiques, il s'avère que lorsque la numération est inférieure à $10 \mathrm{x}$ $106 / \mathrm{ml}$, le pourcentage de transfert d'embryons de classe 3 est significativement abaissé $(18 \%$ versus $37 \% ; \mathrm{P}<0,05)$. De plus, lorsque l'on compare les transferts d'embryons de classe 1 à ceux de classe 3 , le pourcentage de spermatozoïdes anormaux $(48 \% \pm 19 \%$ versus $43 \% \pm 18 \% ; \mathrm{P}<0,05)$ et celui de spermatozoïdes porteurs d'anomalies de la région post acrosomique $(29 \% \pm$ $15 \%$ versus $18 \% \pm 7 \% ; \mathrm{P}<0,05)$ sont supérieurs dans le premier groupe.

Dans le même sens, Janny et Menezo [2] ont montré que le pourcentage d'embryons atteignant le stade blastocyste en coculture était significativement abaissé en cas d'anomalie du sperme.

Ces constatations sont en accord avec les études en clinique humaine de Yovitch et al. [3] et de Ron El et al. [4] qui ont rap-

Tableau 1 : Paramètres des tentatives de FIV en fonction de l'origine de l'infertilité *D'après Parinaud et al. [1].

Stérilités tubaires (626) $156(25 \%)$

$114(18 \%)$

$283(43 \%)$

$229(37 \%)$
Stérilités masculines (84)

$16(19 \%)$

Groupes de morphologie embryonnaire a

1

2

3

$34.1 \pm 4.1$

$33.5 \pm 4$

Age des femmes

$26(31 \%)$

$34(41 \%)$

$34.1 \pm 4.1$

Paramètres spermatiques

numération $\left(10^{6 / \mathrm{ml}}\right)$

mobilité (\%)

$91 \pm 61$

$56 \pm 20$

formes normales $(\%)$

$67 \pm 17$

$44 \pm 55 \mathrm{~b}$

$33 \pm 17 \mathrm{~b}$

$43 \pm 19$ b

Pic E2 (pg/mL)

$1708 \pm 596$

$1681 \pm 579$

Nombre de follicules $\geq 15 \mathrm{~mm}$

$5,5 \pm 2.4$

$5,9 \pm 2.3$

Nombre d'ovocytes

$7,8 \pm 4.1$

$7,9 \pm 4.3$

${ }^{*}$ Moyennes \pm écart-types ; ${ }^{a} P<0,05$ et ${ }^{b} P<0,001$ entre stérilités tubaires et masculines 
porté des développements embryonnaires de mauvaise qualité en cas d'anomalies du sperme. De plus elles corroborent les études expérimentales faites chez l'animal $[5,6]$ qui montrent, après induction d'une hyperthermie testiculo-épididymaire, une augmentation de la mortalité embryonnaire avant même que les altérations de la qualité du sperme soient observables.

Tableau 2 : Formation de blastocystes en fonction de la qualité du sperme, d'après Janny et Menezo [2].

\begin{tabular}{lcc}
\hline Qualité du sperme & Normale & Anormale \\
\hline Nbre de transferts & 144 & 95 \\
$\begin{array}{l}\text { Nbre de cas avec } \\
\text { au moins 1 blastocyste }\end{array}$ & $126(87,5 \%)^{a}$ & $60(63,2 \%)$ \\
Nbre d'embryons & 1397 & 560 \\
Nbre de blastocystes & $621(44,4 \%)^{a}$ & $194(34,6 \%)$ \\
\hline
\end{tabular}

a $P<0,001$ versus sperme anormal

\section{ICSI}

Contrairement à ce qui a été trouvé pour la FIV, à ce jour aucune relation n'a pu être mise en évidence en ICSI entre la qualité du sperme et l'aptitude des embryons à se développer [7, 8]. C'est ainsi que Svalander et al. [9] ont obtenu des taux d'implantation similaires dans les groupes de bonne et mauvaise morphologie spermatique. De plus, lors de l'enquête BLEFCO [10], il est apparu que les taux de grossesse par ponction n'étaient pas du tout influencés par la numération de spermatozoïdes mobiles dans l'éjaculat, même dans les cas d'oligospermie extrême $(<100.000$ spermatozoïdes mobiles/ml), et qu'ils étaient semblables à ceux obtenus en FIV dans les stérilités tubaires à sperme normal. Oehninger et al. [11] ont comparé, dans les tératospermies sévères $(<4 \%$ de formes normales), la qua- lité des embryons obtenus par FIV et par ICSI ; ils ont trouvé que les embryons provenant d'ICSI avaient un score morphologique meilleur et une aptitude à la nidation supérieure.

L'ensemble de ces données suggère donc que le spermatozoïde apporte des éléments essentiels au développement embryonnaire. Ces éléments peuvent être de deux ordres : génétiques et cytoplasmiques, et parmi ces derniers on peut distinguer les facteurs d'activation ovocytaire et le centrosome. Toutefois, l'ICSI semble être capable de court-circuiter, au moins en partie, cette influence du spermatozoïde sur le développement embryonnaire.

\section{FACTEURS SPERMATIQUES CYTOSOLIQUES INFLUENÇANT LE DEVELOPPEMENT EMBRYONNAIRE PRECOCE}

\section{Centrosome}

Le centrosome est le centre cellulaire, organisateur des microtubules, et joue donc un rôle essentiel dans la mise en place du cytosquelette et dans la régulation des divisions cellulaires.

Lors de la spermatogénèse, le centrosome est globalement conservé mais il perd la plupart de la $\gamma$ tubuline [11]. A l'opposé, pendant l'ovogénèse le centrosome disparaît complètement et il ne reste que la $\mathrm{g}$ tubuline [11]. Dans le zygote le centrosome est donc exclusivement d'origine spermatique [12] et le fuseau mitotique des premières mitoses de l'embryon est donc formé uniquement sous l'influence de facteurs paternels [13].

Les defauts de formation du centrosome spermatique pourraient se traduire par une absence de mitose malgré la pénétration du spermatozoïde dans l'ooplasme ou par des mitoses de mauvaise qualité amenant à la formation de débris cellulaires et à une répartition inadéquate des chromosomes 
$[13,14]$. Ces dysfonctionnements du centre cellulaire du spermatozoïde pourraient entraîner la formation d'embryons ayant une faible aptitude au développement. Ainsi, Navar et al. [15] ont montré une relation entre la qualité et la taille du centrosome spermatique et l'aptitude des taureaux à la reproduction. Par ailleurs, Ash et al. [16] ont montré que $25 \%$ des ovocytes n'ayant pas donné d'embryons en FIV humaine sont en réalité fécondés et que la plupart de ces échecs pourraient être dûs à un défaut de centrosome, qui pourrait donc représenter une nouvelle cause de stérilité masculine [17]. Toutefois, l'exploration de la qualité du centrosome est très difficile et fait appel à des techniques non utilisables en routine.

\section{Facteur activateur de l'ovocyte}

Lors de la fusion des gamètes, la pénétration du spermatozoïde entraîne une série d'oscillations du calcium libre dans le cytoplasme ovocytaire $[18,19]$. Ces oscillations entraînent l'activation ovocytaire, c'est-àdire la reprise de la méiose et l'activation de la traduction protéique. Cette mobilisation du calcium intracellulaire est due à un facteur protéique cytosolique thermolabile non spécifique d'espèce [20], appelé oscilline [21], qui agirait en activant une voie de transmission du signal faisant intervenir l'inositol triphosphate. L'injection intracytoplasmique d'oscilline reproduit les oscillations du calcium et induit l'activation ovocytaire. Tesarik et al. [22] ont montré que ces mêmes oscillations se produisaient en ICSI, et que l'augmentation artificielle du calcium libre intra-ovocytaire par le ionophore permettait d'obtenir jusqu'à $90 \%$ d'embryons (versus $32 \%$ chez les témoins) [23]. Un déficit en facteur d'activation pourrait être à l'origine des retards de formations de zygotes, associés à de faibles développements embryonnaires, tels que Ron-El et al. les ont décrits dans les tératospermies [4].

\section{FACTEURS GENETIQUES PATERNELS INFLUENÇANT LE DEVELOPPEMENT EMBRYONNAIRE PRECOCE}

\section{Anomalies chromosomiques}

L'association entre anomalies chromosomiques somatiques et troubles de la spermatogénèse a été largement décrite. C'est ainsi que Bourouillou et al. [24] ont montré qu'environ $7 \%$ des hommes présentant une oligospermie sévère $(<106 / \mathrm{ml})$ et $15 \%$ de ceux ayant une azoospermie étaient porteurs d'une anomalie chromosomique. Chez ces hommes, par ailleurs en bonne santé, le taux d'anomalies chromosomiques est donc environ 15 fois supérieur à celui de la population générale. Parmi ces anomalies, on retrouve essentiellement des anomalies des chromosomes sexuels et des translocations robertsoniennes.

Tableau 3 : Anomalies chromosomiques somatiques en fonction de la numération de spermatozoïdes, d'après Bourrouillou et al. [25].

\begin{tabular}{lcc}
\hline $\begin{array}{l}\text { Numération de } \\
\text { spermatozoides }\end{array}$ & $\begin{array}{c}\text { Nbre de sujets } \\
\text { étudiés }\end{array}$ & $\begin{array}{c}\text { Nbre damomalies } \\
\text { chromosomiques } \\
(\%)\end{array}$ \\
\hline Azoospermie & 440 & $68(15,5)$ \\
$<10 \times 106 / \mathrm{ml}$ & 598 & $39(6,5)$ \\
$10 \times 106 / \mathrm{ml}<<20 \times 106 / \mathrm{ml}$ & 406 & $2(0,5)$ \\
\hline Total & $\mathbf{1 4 4 4}$ & $\mathbf{1 0 9 ( 7 , 5 )}$ \\
\hline
\end{tabular}

De plus, Moosani et al. [26] ont montré que les spermatozoïdes d'hommes infertiles étaient porteurs de plus d'anomalies chromosomiques que les spermatozoïdes d'hommes fertiles.

On peut donc penser que les zygotes issus de spermes anormaux ont un capital génétique altéré. Le génome embryonnaire commençant à s'exprimer, dans l'espèce humaine, à partir du stade 4 cellules, ses anomalies pourraient être responsables de blocages de développement des embryons, tels que l'ont constaté Janny et Ménézo [2]. 


\section{Empreintes génomiques}

Les expériences de reconstruction de zygotes ont clairement montré la complémentarité indispensable des génomes maternels et paternels dans le développement embryonnaire. C'est ainsi que Surani et al. [27] ont montré, chez la souris, que les œufs gynogénotes ( 2 pronuclei femelles) avaient un développement normal de l'embryon lui-même, mais une absence totale de développement du trophoblaste. A l'opposé, les œufs androgénotes ( 2 pronuclei mâles) avaient un développement excessif du trophoblaste et une quasi absence des structures embryonnaires. Ceci suppose que la croissance des annexes de l'œuf est sous la dépendance de gènes exprimés uniquement à partir des chromosomes paternels, les gènes maternels correspondants étant réprimés. Ceci constitue l'empreinte génomique, qui se produit pendant la gamétogénèse, les gènes réprimés étant méthylés. Il semble qu'il en soit de même dans l'espèce humaine. En effet, le pouvoir invasif des tumeurs placentaires est corrélé à la quantité de gènes paternels (Tableau 4).

L'expression génomique préférentielle à été démontrée, dans l'espèce humaine, pour le gène $\mathrm{H} 19$ et pour les gènes codant pour l'IGF2 et pour son récepteur [29]. Ainsi, le gène $\mathrm{H} 19$, qui agirait comme suppresseur de prolifération tumorale, est exprimé abondamment dans le placenta mais pas du

Tableau 4 : Potentiel invasif des tumeurs placentaires en fonction de leur composition génomique. d'après Goshen et al. [28].

\begin{tabular}{lcc}
\hline Histologie & $\begin{array}{c}\text { Rapport } \\
\text { génomique }\end{array}$ & $\begin{array}{c}\text { Potentiel } \\
\text { invasif }\end{array}$ \\
\hline Choriocarcinome & $>2 \mathrm{P}$ & Très élevé \\
Mole complète & $2 \mathrm{P}$ & Elevé \\
Mole partielle & $2 \mathrm{P}: 1 \mathrm{M}$ & Modéré \\
Placenta & $1 \mathrm{P}: 1 \mathrm{M}$ & Normal \\
Triploïdie type 2 & $1 \mathrm{P}: 2 \mathrm{M}$ & Réduit \\
Kyste dermoïde & $2 \mathrm{M}$ & Absent \\
\hline
\end{tabular}

$P$ : génome paternel; $M$ : génome maternel tout dans la môle complète, et serait donc réprimé pendant la spermatogénèse. A l'opposé, le gène de l'TGF2 ne s'exprime qu'à partir des chromosomes paternels.

Il est donc possible que lors d'une spermatogénèse imparfaite il $\mathrm{y}$ ait des anomalies de méthylation de l'ADN conduisant à la sur-expression ou à la sous-expression de certains gènes et donc à des anomalies du développement embryonnaire.

Ces notions prennent une importance plus grande lorsque l'on envisage les techniques d'injection de spermatides rondes (ROSNI) [30]. En effet, lors de la spermiogénèse, de profonds remaniements du noyau se produisent, en particulier le remplacement des histones par les protamines. Il n'est donc pas improbable que ces modifications concernent également les méthylations de l'ADN et donc que les phénomènes d'empreinte génétique soient incomplets au stade spermatide. Toutefois, il faut souligner que cette hypothèse n'a pu être pour l'instant ni prouvée ni infirmée. L'argument des promoteurs de cette technique, basé sur la normalité des enfants nés après ROSNI, est extrêmement fragile dans la mesure où les maladies connues pour être reliées aux phénomènes d'empreinte génétique (tumeur de Wilm, rétinoblastome, ostéosarcome) se révèlent après plusieurs années de vie [31]. Ces données conjuguées à l'absence de recul dans l'expérience animale, doivent conduire, à notre avis, à la plus grande circonspection dans l'utilisation clinique du ROSNI.

\section{CONCLUSION}

La qualité du sperme a donc une influence certaine sur le développement embryonnaire. Toutefois, l'ICSI permet de compenser, au moins en partie, certaines déficiences spermatiques ayant un effet délétère sur la qualité embryonnaire. Comme il paraît peu probable que l'ICSI permette de contourner des anomalies génétiques ou des déficits du 
centrosome, cet effet paternel semble être essentiellement dû au facteur d'activation ovocytaire. En effet, la pénétration de la micropipette dans le cytoplasme pourrait entraîner une décharge massive de calcium intracellulaire, compensant ainsi le déficit spermatique. Dans ce cadre, l'ICSI pallierait non seulement les défauts de fécondation, mais également certaines anomalies embryonnaires induites par les anomalies spermatiques. De plus, l'ICSI permettrait d'éviter les retards de fécondation observés par Ron El et al. [4] dans les tératospermies. A partir de ces données, on peut envisager d'utiliser l'ICSI dans les cas où les embryons obtenus en FIV sont de faible qualité. De plus, l'évaluation de l'oscilline pourrait permettre de diagnostiquer de nouvelles infertilités masculines.

Enfin, il convient de souligner que l'ICSI ne résout certainement pas tous les problèmes d'influence patermelle sur l'embryon, puisque le taux d'implantation n'est au maximum que de $15 \%$ et que les échecs d'implantation sont certainement dûs pour partie à des facteurs paternels. Ces facteurs, en particulier les phénomènes d'empreinte génomique, constituent une voie de recherche essentielle pour la compréhension des mécanismes de développement embryonnaire.

\section{REFERENCES}

1. PARINAUD J., MIEUSSET R., VIEITEZ G., LABAL B., RICHOILLEY G. : Influence of sperm parameters on embryo quality. Fertil. Steril., $1993,60: 888-892$.

2. JANNY L., MENEZO Y.J.R. : Evidence for a strong paternal effect on human preimplantation embryo development and blastocyst formation. Mol. Reprod. Dev., 1994, 38 : 36-42.

3. YOVITCH J.L., STANGER J.D. : The limitations of in vitro fertilization from males with severe oligospermia and abnormal sperm morphology. J. In Vitro Fert. Embryo Transf., 1984, $1: 172-9$.

4. RON-EL R., NACHUM H., HERMAN A., GOLAN A., CASPI E., SOFFER E. : Delayed fertilization and poor embryonic development associated with impaired semen quality. Fertil. Steril., 1991, 55 : 338-44.
5. SETCHELL B.P., D'OCCHIO M.J., HALL M.J., LAURIE M.S., TUCKER M.J., ZUPP J.L. : Is embryonic mortality increased in normal females mated to subfertile males? J. Reprod. Fertil., $1988,82: 567-74$.

6. MIEUSSET R., QUINTA CASARES P., SANCHEZ PARTIDA L.G., SOWERBUTS S.F., ZUPP J.L., SETCHELL B.P. : Effects of heating the testes and epididymides of ram by scrotal insulation on ferti. lity and embryonic mortality in ewes inseminated with frozen sperm. J. Reprod. Fertil,, 1992, 94 : $337-43$.

7. PALERMO G., JORIS H., DERDE M-P., CAMUS M., DEVROEY P., VAN STEIRTEGHEM A.C. : Sperm charactersistics and outcome of human assisted fertilization by subzonal insemination and intracytoplasmic sperm injection. Fertil. Steril., $1993,59: 826-35$.

8. MANSOUR R.T.,"ABOULGHAR M.A., SEROUR G.I., AMIN Y.M., RAMZI A.M. : The effect of sperm parameters on the outcome of intracytoplasmic sperm injection. Fertil. Steril., 1995, 64 : 982-6.

9. SVALANDER P., JAKOBSON A-H., FORSBERG A-S., BENGTSSON A-C., WIKLAND M. : The outcome of intracytoplasmic sperm injection is unrelated to "strict" sperm morphology. Hum. Reprod., 1996, $11: 1019-22$.

10. COHEN-BACRIE P., HAMAMAH S., PARINAUD J., ZERAH S. : La micro-injection en France : rapport de l'enquête multicentrique des BLEFCO. Contracept. Fertil. Sex., 1995, $23: 477-80$.

11. OEHNINGER S., KRUGER T.F., SIMON T., JONES D., MAYER J., LANZENDORF S., et al. : A comparative analysis of embryo implantation potential in patients with severe teratozoospermie undergoing in-vitro fertilization with a high insemination concentration or intracytoplasmic sperm injection. Hum. Reprod. 1996, 11 : 1086-9.

12. SCHATTEN G. : The centrosome and its mode of inheritance : the reduction of the centrosome during gametogenesis and its restoration during fertilization. Dev. Biol., 1994, 165 : 299-335.

13. PALERMO G., MUNNE S., COHEN J. : The human zygote inherits its mitotic potential from the male gamete. Hum. Reprod., 1994, $9: 1220-5$.

14. SIMERLY C., WU G-J., ZORAN S., ORD T., RAWLINS R., JONES J., et al. : The paternal inheritance of the centrosome, the cell's microtubulesorganizing center, in humans, and the implications for infertility. Nature Medicine, 1995, 1 : 47-52.

15. NAVARA C., FIRST N., SCHATTEN G. : Individual bulls affect sperm aster size and quality : relationship between the sperm centrosome and development. Mol. Biol. Cell, 1993, $4: 828$. 
16. ASCH R., SIMERLY C., ORD T., ORD V.A., SCHATTEN $G$. : The stages at which human fertilization arrests : microtubule and chromosome configurations in inseminated oocytes which failed to complete fertilization and development in humans. Hum. Reprod., 1995, 10 : 1897-1906.

17. VAN BLERKOM J. : Sperm centrosome dysfunction : a possible new class of male factor infertility in the human. Mol. Hum. Reprod., 1996, 2 : 349-54.

18. TAYLOR C.T., LAWRENCE Y.M., KINGSLAND C.R., BILJAN M.M., CUTHBERTSON K.S.R. : Oscillations in intracellular free calcium induced by spermatozoa in human oocytes at fertilization. Hum. Reprod., 1993, 8 : 2174-9.

19. SWANN K., LAWRENCE Y. : How and why spermatozoa cause calcium oscillations in mammalian oocyte. Hum. Reprod., 1996, 11 : 388-90.

20. DOZORTSEV D., RYBOUCHKIN A., DE SUTTER P., QIAN C., DHONT M. : Human oocyte activation following intracytoplasmic injection : the role of the sperm cell. Hum. Reprod., 1995, $10: 3-407$.

21. PARRINGTON J., SWANN K, SHVECHENKO V.I. : Calcium oscillations in mammalian oocytes triggered by a soluble sperm protein. Nature, 1996, $379: 364-8$.

22. TESARIK J., SOUSA M., TESTART J. : Human oocyte activation after intracytoplasmic sperm injection. Hum. Reprod., 1994, $9: 511-8$.

23. TESARIK J., SOUSA M. : More than $90 \%$ fertilization rates after intracytoplasmic sperm injection and artificial induction of oocyte activation with calcium ionophore. Fertil. Steril., 1995, 63 : 343-9.

24. BOUROUILLOU G., DASTUGUE N., COLOMBIES P. : Chromosome studies in 952 infertile male with a sperm count below 10 millions $/ \mathrm{ml}$. Hum. Genet., 1985, $71: 366$.

25. BOURROUIlloU G., MANSAT A., CALVAS P., PONTONNIER F., COLOMBIES P. : Anomalies chromosomiques et infertilité masculine. Etude de 1444 sujets. Bulletin de l'Association des Anatomistes, 1987, $71: 29-31$.

26. MOOSANI N., PATTINSON N., CARTER M.D., COX D.M., RADEMAKER A.W., MARTIN R.H. : Chromosomal analysis of sperm from men with idiopathic infertility using sperm karyotyping and fluorescence in situ hybridization. Fertil. Steril., 1995, 64 : 811-7.

27. SURANI M.A.H., BARTON S.C., NORRIS M.L. : Experimental reconstruction of mouse eggs and embryos: an analysis of mammalian development. Biol. Reprod., 1987, 36 : 1-16.

28. GOSHEN R., BEN-RAFAEL Z., GONIK B., LUSTIG O., TANNOS V., DE-GROOT N., et al. : The role of genomic imprinting in implantation. Fertil. Steril., 1994, $62:$ 903-10.
29. DE-GROOT N., HOCHBERG A. : Gene imprinting during placental and embryonic development. $\mathrm{Mol}$. Reprod. Dev., 1993, 36 : 390-406.

30. TESARIK, J., ROLET, F., BRAMI, C., et al. : Spermatid injection into human oocytes. II. Clinical application in the treatment of infertility due to non-obstructive azoospermia. Hum. Reprod., 1996, 11 : 780-783.

31. FEINBERG A.P. : Genomic imprinting and gene activation in cancer. Nature Genet., 1993, 4 : 110-113.

\section{ABSTRACT \\ Influence of sperm factors on early human development}

\section{J. Parinaud}

The influence of sperm factors on early human embryonic development has been shown through the production of lower quality embryos in case of in vitro fertilization (IVF) for male infertility when compared to tubal infertility. Numerous factors may be implied in this effect. Indeed, zygotic centrosome, cellular organizer having a key role in mitosis, is exclusively from paternal origin and some IVF failures have correlated to centrosome deficiencies. A sperm cytosolic protein, oscillin, induces oocyte activation after sperm-egg fusion and could be responsible of clivage retardations observed in sperm abnormalities. Paternal chromosomal abnormalities, shown in as much as $8 \%$ of severe oligospermia, could lead to development blockage of the embryos. Last, genome imprinting during spermatogenesis, could be impaired in testicular dysfunctions, and thus the embryos could have low developmental abilities. However, it must be pointed out that no influence of sperm quality has been found using intracytoplasmic sperm injection (ICSI) procedures. Thus we can postulate that this technique alleviate the problem found with IVF. Thus the factor involved in the paternal effect on early embryogenesis is likely oocyte activation.

Key words : Spermatozoa, embryo, centrosome, oscillin, genetics. 